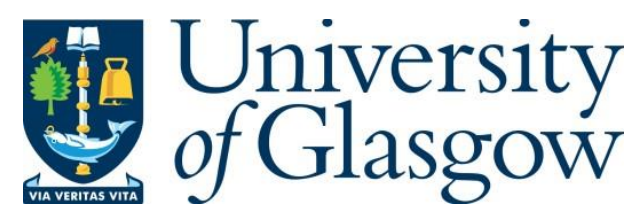

Mason, A., Gerasimidis, K., Iljuhhina, J., Laird, S., Munro, J., Gaya, D. R., Russell, R. K. and Ahmed, S. F. (2017) Long-term skeletal disproportion in childhood-onset Crohn's disease. Hormone Research in Paediatrics, (doi:10.1159/000485185).

There may be differences between this version and the published version. You are advised to consult the publisher's version if you wish to cite from it.

http://eprints.gla.ac.uk/153446/

Deposited on: 12 December 2017

Enlighten - Research publications by members of the University of Glasgow http://eprints.gla.ac.uk 


\section{Long-term Skeletal Disproportion In Childhood-Onset Crohn's Disease}

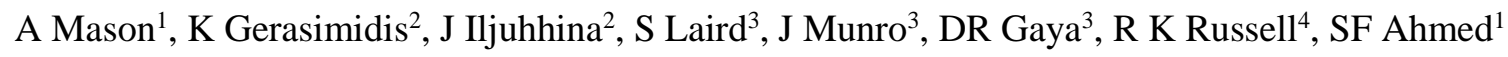

${ }^{1}$ Developmental Endocrinology Research Group, School of Medicine, College of Medical, Veterinary and Life Sciences, University of Glasgow, Royal Hospital for Children, Glasgow, UK

${ }^{2}$ Human Nutrition, School of Medicine, College of Medical, Veterinary and Life Sciences, University of Glasgow, Glasgow Royal Hospital, Glasgow, UK

${ }^{3}$ Department of Gastroenterology, Glasgow Royal Infirmary, Glasgow, UK

${ }^{4}$ Department of Paediatric Gastroenterology Hepatology and Nutrition, Royal Hospital for Children, Glasgow, UK

\section{Address for correspondence}

Dr Avril Mason

Developmental Endocrinology Research Group, University of Glasgow

Royal Hospital for Children

Glasgow G51 4TF, United Kingdom

Tel +44 (141) 2010000

Fax +44 (141) 2010837

$\underline{\text { AvrilMason@nhs.net }}$

ESPE membership 121426

Word Count (Excluding Abstract, Legends \& References): 1098

Short Title: Body Disproportion in CD

Keywords: Chronic Diseases, Inflammatory Bowel Disease, Adult Height, Inflammation, Stature 


\begin{abstract}
Background

It is unclear whether Crohn's disease (CD) is associated with skeletal disproportion in adulthood.
\end{abstract}

\title{
Methods
}

Height (Ht), sitting height (SHt) and leg length was studied in 44 children (C) (M:22), 23 adults (M:10) with childhood-onset (CO-CD) and 26 adults (M:9) with adult-onset (AO) CD with a median (range) age of 13.7 yrs $(10,17.3), 21.5 \mathrm{yrs}(18,32)$ and 31.0 yrs $(18,40)$, respectively.

\section{Results}

Adults with CO-CD had a median Ht SDS of -0.9 $(-2.3,0.0)$ compared to $0.6(-0.8,1.0)$ in those with AO-CD $(\mathrm{p}<0.05)$. Compare to normal, men, but not women, with CO-CD also had lower median SHt SDS at -1.1 $(2.5,-0.5)(\mathrm{p}<0.05)$. The expected positive association that is normally found between leg length and SHt SDS was not evident in the adults with CO-CD.

\section{Conclusion}

Short stature in adults with childhood-onset CD is more pronounced in men and may be associated with poor spinal growth.

Word count 148 


\section{Introduction}

Crohn's Disease (CD) often presents during early adolescence. Clinical presentation is commonly associated with short stature and, historically, delayed puberty. Adults with childhood-onset (CO) CD (CO-CD) are shorter than expected for their mid-parental height [1]. Although pubertal delay does not seem to be a major concern in children on contemporary therapy, affected children and adolescents with CD continue to remain shorter than the reference population [2] and exhibit suboptimal growth during puberty [3]. We performed a cross-sectional study of height and skeletal proportions in children with $\mathrm{CD}$ as well as adults with $\mathrm{CO}-\mathrm{CD}$ and AO-CD to further explore the contribution of pubertal growth. 


\section{Patients \& Methods}

Eligible participants were children less than 18 yrs old with CD attending the, Royal Hospital for Children, Glasgow and young adults between the age of 18 and $40 \mathrm{yr}$ attending the Glasgow Royal Infirmary, Glasgow. The latter, were divided into two groups: CO-CD: onset $<18 \mathrm{yr}$, AO-CD: disease onset $\geq 18 \mathrm{yr}$. Prepubertal onset of symptoms was defined using chronological age at onset of symptoms as $<13$ years for males and <11 years for females [2]. Patients with known endocrinopathies or other co-morbidities that may affect growth or skeletal development were excluded from recruitment. Clinical details were collected from case notes and face to face interview. Anthropometric data were obtained using standard protocols. Height (Ht) and Sitting Ht (SHt) were measured with a wall-mounted stadiometer with Leg length (LL) calculated as the difference between the $\mathrm{Ht}$ and SHt. Parental heights were measured for the children with $\mathrm{CD}$ and were self-reported by the adults with CD; 2 adults with CO-CD and 3 with AO-CD could not recall parental heights.

All data were expressed as median (range) and analysed (Minitab 16, Coventry, UK). Ht, SHt and leg length (LL) were expressed as standard deviation scores (SDS) and short stature was defined as Ht SDS $\leq-2$. A deviation from target/mid-parental Ht SDS was defined as the difference between the target and patient's actual Ht SDS. One sample t-test was used to compare height measurements against the reference UK population data. Mann-Whitney $U$ test was used for inter-group comparisons and Pearson correlation was used to assess associations between continuous variables. Statistical significance was set at a $p<0.05$.

The study was approved by the West of Scotland Research Ethics Committee (10/S0701/66) and each participant provided written consent prior to study enrollment. 


\section{Results}

\section{Participants Characteristics}

Participant characteristics are presented in Table 1 . Of the 92 patients recruited, 44 were children with CD $(\mathrm{M}, 22), 26$ were adults with AO-CD (M,9) and 23 were adults with CO-CD (M,10). Pre-pubertal onset of disease was noted in 29/44 (65.9\%) children, and in 10/23 (43.4\%) adults with CO-CD (Table 1).

\section{Height and skeletal proportions in children with $C D$}

There was no significant difference in Ht, SHt, and LL SDS between girls and boys (Table 1). Compared to the UK 1990 reference data, boys but not girls had a median Ht deficit of 0.63 SD (Table 1). Whilst both sex groups also had a lower SHt SDS, boys with CD also had a lower LL SDS (Table 1). There was a positive correlation between SHt and LL SDS in children with pre-pubertal $(r=0.54 ; \mathrm{p}=0.003)$ and pubertal $(\mathrm{r}=0.84$; $\mathrm{p}<0.001$ ) onset of CD (Figure 1). The five children with greatest disproportion, either LL SDS $\leq-2$ or a SHt SDS $\leq-2$, were amongst those with the lowest Ht SDS. The four (M,3) with low LL SDS but normal SHt SDS had pre-pubertal onset of CD and the one girl with low SHt SDS but normal LL SDS was diagnosed in mid-puberty.

\section{Final height and skeletal proportions in adults with adult and childhood-onset CD}

Median Ht SDS and SHt SDS in men with CO-CD was lower than that of AO-CD (Table 1). In addition, men with CO-CD also showed a larger deviation from target Ht. However, these men did not show a significant difference in LL SDS (Table 1). In women there was no significant difference in Ht, SHt, LL SDS and deviation from target $\mathrm{Ht}$ between participants with CO-CD or AO-CD (Table 1). The positive association which was evident between SHt and LL SDS for adults with AO-CD ( $r=0.47 ; \mathrm{p}=0.016)$ was not present in those with CO-CD $(r=0.09 ; \mathrm{p}=0.677)$ (Figure 1). Notably two patients each had LL SDS $\leq-2$ or low SHt SDS $\leq-2$. These four represented those with the lowest Ht SDS within the entire group, all were adults with CO-CD (Figure 1). The two patients with low SHt SDS but normal LL SDS (patient A and B) were diagnosed in mid-puberty and the two with low LL SDS but normal SHt SDS (patient C and D) had pre-pubertal onset of CD. In the adults with CO-CD or AO-CD, there was no association of Ht or SHt SDS with the age at onset of disease, disease duration, Montreal classification of disease or the use of biologic therapy as a marker of disease severity. 


\begin{tabular}{|c|c|c|c|c|c|c|c|c|c|c|c|c|}
\hline & \multirow{2}{*}{\multicolumn{2}{|c|}{$\begin{array}{l}\text { Female } \\
\text { C }(n=22)\end{array}$}} & \multirow{2}{*}{\multicolumn{2}{|c|}{$\begin{array}{c}\text { Male } \\
C(n=22)\end{array}$}} & \multicolumn{4}{|c|}{ Female } & \multicolumn{4}{|c|}{ Male } \\
\hline & & & & & \multicolumn{2}{|c|}{$\operatorname{CO}-\mathrm{CD}(\mathrm{n}=13)$} & \multicolumn{2}{|c|}{ AO-CD $(n=17)$} & \multicolumn{2}{|c|}{ CO-CD $(n=10)$} & \multicolumn{2}{|c|}{$\mathrm{AO}-\mathrm{CD}(\mathrm{n}=9)$} \\
\hline & Median & Range/\% & Median & Range/\% & Median & Range/\% & Median & Range/\% & Median & Range/\% & Median & Range/\% \\
\hline Age at diagnosis, (y) & 10.9 & $6: 15.5$ & 11.1 & $2: 14.2$ & 14.0 & $10: 17$ & $24.0 \dagger$ & $20: 37$ & 12.0 & $7: 15$ & $22.0 \dagger$ & $19: 27$ \\
\hline Age at recruitment, $(y)$ & 13.9 & 10:17 & 13.4 & 10:17.3 & 23.0 & $18: 30$ & $32.0 \dagger$ & $22: 40$ & 20.0 & $18: 32$ & $30.0 \dagger$ & $24: 39$ \\
\hline \multicolumn{13}{|l|}{ Localisation: } \\
\hline Ileal & & & & & 1 & & 5 & & 3 & & 2 & \\
\hline Colonic & 4 & & 4 & & 3 & & 2 & & 2 & & & \\
\hline Ileocolonic & 15 & & 16 & & 7 & & 9 & & 4 & & 6 & \\
\hline Isolated upper & & & & & 1 & & & & & & 1 & \\
\hline Ileocolonic + upper & 3 & & 2 & & 1 & & & & 1 & & & \\
\hline Perianal & 2 & & & & & & 1 & & & & & \\
\hline \multicolumn{13}{|l|}{ Treatment: } \\
\hline Surgery & 0 & & 0 & & 5 & $38 \%$ & 13 & $76 \%$ & 6 & $67 \%$ & 5 & $56 \%$ \\
\hline Biologics & 2 & $9 \%$ & 1 & $5 \%$ & 5 & $38 \%$ & 9 & $53 \%$ & 7 & $78 \%$ & 6 & $75 \%$ \\
\hline Thiopurines & 12 & $55 \%$ & 9 & $41 \%$ & 5 & $38 \%$ & 9 & $53 \%$ & 1 & $11 \%$ & 6 & $75 \%$ \\
\hline Methotrexate & 6 & $27 \%$ & 9 & $41 \%$ & 4 & $31 \%$ & 2 & $12 \%$ & 2 & $22 \%$ & 0 & $0 \%$ \\
\hline Aminosalicylates & 7 & $32 \%$ & 9 & $41 \%$ & 6 & $46 \%$ & 3 & $18 \%$ & 2 & $22 \%$ & 3 & $33 \%$ \\
\hline Oral steroids & 2 & $9 \%$ & 3 & $14 \%$ & 4 & $31 \%$ & 6 & $35 \%$ & 0 & $0 \%$ & 1 & $12 \%$ \\
\hline \multicolumn{13}{|l|}{ Anthropometry: } \\
\hline Height SDS & -0.1 & $-2.5: 2.0$ & -0.2 & $-2.6: 1.5$ & -0.4 & $-1.7: 2.1$ & -0.1 & $-1.3: 1.8$ & $-0.9^{*}$ & $-2.3: 0.0$ & $0.6 \dagger$ & $-0.8: 1.0$ \\
\hline Deviation from target height $(\mathrm{SDS})^{\S}$ & -0.2 & $-2.5: 1.3$ & -0.2 & $-2.4: 2.4$ & 0.1 & $-1.5: 1.0$ & -0.2 & $-1.1: 1.6$ & 0.3 & $-0.5: 1.5$ & $-0.5 \dagger$ & $-1.5: 1.0$ \\
\hline Sitting height SDS & $-0.4^{*}$ & $-3.0: 1.8$ & $-0.5^{*}$ & $-2.2: 1.0$ & -0.6 & $-2.8: 1.8$ & $-0.7^{*}$ & $-1.6: 0.6$ & $-1.1 *$ & $-2.5:-0.5$ & $0.2 \uparrow$ & $-0.7: 0.8$ \\
\hline Leg length SDS & 0.0 & $-2.7: 1.6$ & $-0.3 *$ & $-3.0: 1.7$ & -0.1 & $-1.5: 1.6$ & 0.3 & $-1.6: 1.8$ & -0.3 & $-3.1: 1.4$ & 0.4 & $-0.8: 0.9$ \\
\hline
\end{tabular}




\section{Discussion}

In contemporary cohorts, boys with $\mathrm{CD}$ are more likely to have an abnormality of pubertal growth despite satisfactory entry and progress through puberty [3], and are therefore, at risk of a lower final adult height. Poor growth, and an abnormality of the GH-IGF1 axis, are evident during periods of active disease and may be related to multiple factors including malnutrition and an elevation in pro-inflammatory cytokines [4]. In accordance with previous research [1], the current study suggests that short stature in childhood-onset CD may persist into adulthood. However, the current study also shows that the growth restriction is more likely in boys and may be linked more specifically to a reduction in spinal length. This finding of relative skeletal disproportion is further supported by the lack of association between LL and SHt in adults with CO-CD. Interestingly the four adults displaying the greatest disproportion had the lowest Ht SDS and all had CO-CD. A previous history of pubertal delay or abnormal pubertal growth provides a possible explanation for skeletal disproportion in adulthood [5] and may explain the greater height restriction as well as disproportion seen in boys with $\mathrm{CD}$ and men with $\mathrm{CO}-\mathrm{CD}$. There is also the possibility that the reduced sitting height seen in this study may be related to subclinical vertebral fractures or abnormal vertebral morphology, which is becoming increasingly recognised in young people with chronic inflammatory disease as well as those who require systemic glucocorticoids [6]. Prospective studies would require longitudinal assessment of puberty and radiological assessment of vertebral morphology.

In summary, by showing that short stature in adults with CO-CD is more marked in boys and may be associated with skeletal disproportion, this study provides further mechanistic insight into the effect of childhood chronic disease on growth and skeletal development. 


\section{Acknowledgements}

AM was supported by the University of Glasgow and the Glasgow Children's Hospital Charity.

\section{Conflicts of interest}

The authors do not have any conflict of interest.

Table I: Demographics, disease characteristics and descriptors of height in children (C) and adults with childhood (CO-CD) and adulthood (AO-CD) onset Crohn's disease. SDS - Standard deviation score; * $\mathrm{p}<0.05$ compared with UK 1990; $\uparrow \mathrm{p}<0.05$ ACO vs AAO for same gender; $\$ p<0.05$ between genders for the same time of CD onset; ${ }^{\S}$ Difference between target (mid parental) and actual standing height SDS.

Figure 1: Sitting Height SDS vs Leg Length SDS in Adults with Childhood (CO-CD) and adulthood (AOCD) onset Crohn's Disease. Patient A: Male, HtSDS: -1.87, pubertal onset; Patient B: Female, HtSDS: -1.73, pubertal onset; Patient C: Male, HtSDS: -2.11, pre-pubertal onset; Patient D: Male, HtSDS:- -2.26, prepubertal onset. SDS - Standard deviation score: Ht-height. 


\section{References}

1. Sawczenko A, Ballinger AB, Croft NM, Sanderson IR, Savage MO: Adult height in patients with early onset of Crohn's disease. Gut 2003;52:454-455.

2. Malik S, Mason A, Bakhshi A, Young D, Bishop J, Garrick V, McGrogan, Russell RK, Ahmed SF: Growth in children receiving contemporary disease specific therapy for Crohn's disease. Arch Dis Child 2012;97:698-703.

3. Mason A, Malik S, Russell RK, Bishop J, McGrogan P, Ahmed SF: Impact of inflammatory bowel disease on pubertal growth. Horm Res Paediatr 2011;76:293-9.

4. Wong SC, Dobie R, Altowati MA, Werther GA, Farquharson C, Ahmed SF: Growth and the growth hormone-insulin like growth factor 1 axis in children with chronic inflammation: current evidence, gaps in knowledge, and future directions. Endocr Rev 2015;37:62-110.

5. Albanese A, Stanhope R: Does constitutional delayed puberty cause segmental disproportion and short stature? European Journal of Pediatrics 1993;152:293-296.

6. Kyriakou A, Shepherd S, Mason A, Ahmed SF: Prevalence of vertebral fractures in children with suspected osteoporosis. J Pediatr2016;179:219-225. 


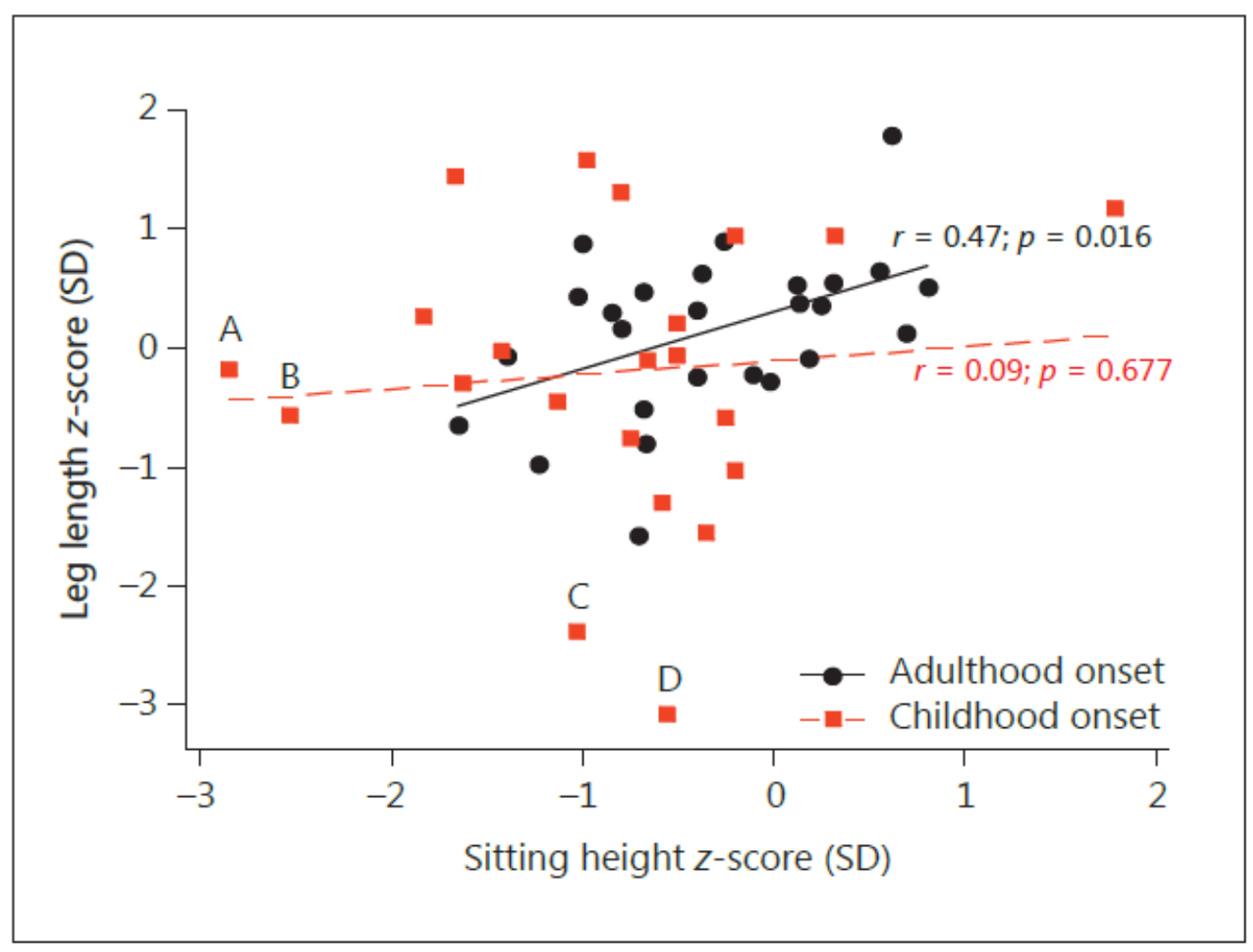

Fig. 1. Sitting height SDS versus leg length SDS in adults with childhood- and adult-onset Crohn's disease. Patient A: male, Ht SDS -1.87 , pubertal onset; patient B: female, Ht SDS -1.73 , pubertal onset; patient C: male, Ht SDS -2.11, pre-pubertal onset; patient D: male, Ht SDS -2.26, pre-pubertal onset. SDS, standard deviation score; Ht, height. 